\title{
RESEARCH
}

Open Access

\section{LncRNA NORAD promotes bone marrow stem cell differentiation and proliferation by targeting miR-26a-5p in steroid-induced osteonecrosis of the femoral head}

\author{
Dapeng Fu ${ }^{1 *} \mathbb{D}$, Sheng Yang ${ }^{1}$, Jianmin Lu' ${ }^{1}$ Haoyi Lian ${ }^{1}$ and Kairong Qin ${ }^{2}$
}

\begin{abstract}
Background: Steroid-induced osteonecrosis of the femoral head (SONFH) is a devastating orthopedic disease, which seriously affects the quality of life of patients. The study aims to investigate the effects of LnCRNA NORAD on SONFH.

Methods: Human bone marrow-derived mesenchymal stem cells (hBMSCs) were isolated from the proximal femur of patients during routine orthopedic surgery and then cultured with dexamethasone (Dex) and transfected with NORAD overexpression vector, siRNA-NORAD and miR-26a-5p mimics. The mRNA expression of NORAD, miR-26a-5p, OPG, RANK, and RANKL was detected by RT-qPCR. Cell proliferation and apoptosis was measured by CCK-8 assay and flow cytometry, respectively. The protein expression of RUNX2, OPG, RANK, and RANKL was detected by western blot. The dual-luciferase reporter gene assay was performed to confirm the binding between NORAD and miR-26a-5p.

Results: NORAD expression was downregulated in SONFH tissues, while miR-26a-5p expression was upregulated. Overexpression of NORAD improved DEX-induced inhibition of proliferation and differentiation, and promotion of apoptosis in hBMSCs, while knockdown of NORAD led to the opposite results. Moreover, NORAD improved DEXinduced inhibition of proliferation and differentiation, and promotion of apoptosis by regulation of miR-26a-5p in hBMSCs.

Conclusions: NORAD expression was downregulated in SONFH tissues, while miR-26a-5p expression was upregulated. NORAD improved DEX-induced inhibition of proliferation and differentiation, and promotion of apoptosis by regulation of miR-26a-5p in hBMSCs.
\end{abstract}

Keywords: Steroid-induced osteonecrosis of the femoral head, LncRNA NORAD, miR-26a-5p, OPG/RANK/ RANK L pathway, Proliferation

\footnotetext{
* Correspondence: dapeng996@126.com

'Department of Orthopaedics, Affiliated Zhongshan Hospital of Dalian University, Dalian 116001, Liaoning, People's Republic of China

Full list of author information is available at the end of the article
}

(c) The Author(s). 2021 Open Access This article is licensed under a Creative Commons Attribution 4.0 International License, which permits use, sharing, adaptation, distribution and reproduction in any medium or format, as long as you give appropriate credit to the original author(s) and the source, provide a link to the Creative Commons licence, and indicate if changes were made. The images or other third party material in this article are included in the article's Creative Commons licence, unless indicated otherwise in a credit line to the material. If material is not included in the article's Creative Commons licence and your intended use is not permitted by statutory regulation or exceeds the permitted use, you will need to obtain permission directly from the copyright holder. To view a copy of this licence, visit http://creativecommons.org/licenses/by/4.0/. The Creative Commons Public Domain Dedication waiver (http://creativecommons.org/publicdomain/zero/1.0/) applies to the data made available in this article, unless otherwise stated in a credit line to the data. 


\section{Background}

Osteonecrosis of the femoral head (ONFH) is characterized by the collapse of the femoral head due to bone cells and bone marrow to be necrotic $[1,2]$. At present, glucocorticoid (GC) has been commonly used to treat rheumatic, autoimmune, inflammation-dependent diseases and hematopoietic system diseases [3]. However, the usage of GC is one of the most common causes of $\mathrm{ONFH}$ [4]. Clinical observation confirmed that most patients who were treated with high-dose GC appeared clinical symptoms of osteonecrosis within 2 years [1]. Moreover, glucocorticoid-induced osteonecrosis of the femoral head (GIOFH) usually involves young adults and most patients with GIOFH require surgery, which seriously affects the quality of life of patients $[5,6]$. However, the pathogenesis of steroid-induced osteonecrosis of the femoral head (SONFH) is unclear.

GC can inhibit the bone formation by affecting bone cell [3]. One study has been indicated that the change of osteogenic differentiation ability of mesenchymal stem cells (MSCs) is the cause of the imbalance of necrosis and bone regeneration, which is a key factor in the pathogenesis of non-traumatic ONFH [7]. Bone marrow mesenchymal stem cells (BMSCs) are a group of pluripotent stem cells with multidirectional differentiation potential capabilities [8, 9]. Osteoblast, chondroblast, and adipocyte can be derived from BMSCs, which may further secrete growth factors to promote tissue regeneration [10]. Human bone marrow-derived mesenchymal stem cells (hBMSCs) are characterized by a negative phenotype of hematopoietic lineage, which are derived from the human bone marrow cavity [1]. Recent studies have shown that the occurrence and development of many orthopedic diseases are closely related to hBMSCs $[7,11]$. Therefore, the analysis of the potential mechanisms of aberrant osteogenic differentiation of BMSCs may play an important role in the treatment of SONFH.

Long noncoding RNAs (lncRNAs) are RNAs that lack the ability of protein coding and their length are more than 200 nucleotides [12]. In recent years, numerous studies have demonstrated that lncRNAs play a very important role in cell proliferation, osteogenic differentiation, apoptosis, and autophagy [13-15]. LncRNA NORAD, as a long noncoding RNA activated by DNA damage, expresses abnormally in many kinds of tumors and serves as an oncogene [16-18]. Xu et al. [19] have reported that NORAD promote the proliferation, invasion, migration, and EMT of ovarian cancer cells. Wang et al. [20] have found that knockdown of NORAD suppressed osteosarcoma cell proliferation and in vivo explant growth. In addition, Tao et al. [21] have revealed that NOARD can inhibit the apoptosis of gastric cancer cells by down-regulating caspase-3. Additionally, it also has been reported that NORAD attenuates endothelial cell apoptosis [22]. Micro RNAs (miRNAs), as noncoding single-stranded RNA, are involved in proliferation, apoptosis, differentiation, and so on [23-25]. Studies have revealed that the interaction of lncRNAs and miRNAs can affect the osteogenic differentiation of BMSCs [26]. Li et al. [27] have found that upregulation of miR-26a-5p inhibits osteogenesis and downregulation of miR-26a-5p promotes osteogenesis. However, the relationship between NORAD and miR-26a-5p in SONFH is not reported.

In this study, we detected the expression of NORAD and miR-26a-5p in SONFH tissues. In addition, we explored the biological effects of NORAD and miR-26a-5p on cell proliferation, apoptosis, and OPG/RANK/RANKL pathway in hBMSCs. Our findings may provide a new target direction for the treatment and diagnosis of SONFH.

\section{Material and methods \\ Subjects and specimen collection}

Bone marrow samples were obtained from 20 patients with SONFH as well as 20 patients with femoral neck fracture who underwent surgeries between April 2018 and March 2020 at our hospital. According to the Steinberg or University of Pennsylvania system, diagnosis of SONFH was proved by preoperative radiographs and magnetic resonance image (MRI). All patients with SONFH who had a history of taking a greater than 1800 mg of GCs or a long-term glucocorticoid therapy for more than 4 weeks were included, and patients concurrent with cardiovascular diseases, congenital diseases, or tumor-related diseases were excluded in the present study. In addition, patients with femoral neck fracture have no history of GC therapy. All participants were signed written informed consent, and our study was approved by the ethics committee of our hospital.

\section{Isolation and culture of hBMSCs}

Bone marrow aspirates $(10 \mathrm{ml})$ were obtained from the proximal femur of patients during routine orthopedic surgery. The aspirates were resuspended in phosphatebuffered saline (PBS), and then, the cell suspension was injected into a centrifuge tube containing an equal volume of lymphocyte separation solution. After centrifugation for $30 \mathrm{~min}$ at $2000 \mathrm{r} / \mathrm{min}$, the mononuclear cells in the white layer were collected and cultured in lowglucose Dulbecco's modified Eagle's medium (DMEMLG, Gibco, Rockville, MD, USA) with $10 \%$ fetal bovine serum (FBS, Gibco) and Penicillin-Streptomycin solution (Invitrogen, Carlsbad, CA, USA) at $37^{\circ} \mathrm{C}$ in $5 \% \mathrm{CO}_{2}$. For inhibition of differentiation, the cells were treated with different concentration of dexamethasone (DEX;10 ${ }^{-8}$, $10^{-7}$, and $10^{-6} \mathrm{M}$; Sigma). 


\section{Cell transfection}

BMSCs were divided into control (without treatment), Dex (BMSCs were treated with $10^{-6}$ Dex), Dex+Vector [BMSCs were treated with $10^{-6}$ Dex and transfected with negative control of NORAD (Vector)], Dex+NORAD (BMSCs were treated with $10^{-6}$ Dex and transfected with NORAD overexpression vector), Dex+si-NC [BMSCs were treated with $10^{-6}$ Dex and transfected with negative control of NORAD (si-NC)], Dex+si1NORAD (BMSCs were treated with $10^{-6}$ Dex and transfected with siRNA1-NORAD), Dex+Vector+mimics NC (BMSCs were treated with $10^{-6}$ Dex and transfected with Vector and mimics NC), and Dex+NORAD+miR26a-5p mimics (BMSCs were treated with $10^{-6}$ Dex and transfected with NORAD overexpression vector and miR-26a-5p mimics) group. NORAD, si-NORAD, miR26a-5p mimics, and corresponding negative control were synthesized by GenePharma (Shanghai, China). The transfection was performed by Lipofectamine 2000 (Invitrogen) according to the manufacturer's instructions. Reverse transcription-quantitative polymerase chain reaction (RT-qPCR) was used to determine the transfection efficiency.

\section{RT-qPCR assay}

According to manufacturer's protocol, TRIzol reagent (Invitrogen) was used to extract total RNA from tissues and cells. Total RNA was taken for reverse transcription of cDNA by a PrimeScript RT-PCR Kit (Takara, Dalian, China). Subsequently, RT-PCR was performed by the SYBR Green PCR kit (Applied Biosystems, Shanghai, China) on ABI 7500 real-time PCR system (Applied Biosystems). The relative mRNA expression was calculated by $2^{-\Delta \Delta C t}$ method, and $\beta$-actin or U6 was used as the internal reference. Primer sequences (Table 1) were synthesized by Invitrogen.

\section{Western blot analysis}

Radioimmunoprecipitation assay (RIPA) buffer (SigmaAldrich, St. Louis, MO, USA) was used to extract total protein from BMSCs. BCA assay (Beyotime, Nanjing, China) was used to detect the total protein concentration. Equal quantities of protein $(30 \mu \mathrm{g})$ were subjected to $10 \%$ sodium dodecyl sulphate-polyacrylamide gel electrophoresis and transferred onto polyvinylidene fluoride membranes (Millipore, Billerica, MA,USA). After being blocked with $5 \%$ fat-free milk, the membranes were incubated with primary antibody (RUNX2, ab192256; OPG, ab73400; RANK, ab200369; RANKL, ab9957; GAPDH, ab181602. 1: 1000. Abcam, Cambridge, MA, USA) at $4{ }^{\circ} \mathrm{C}$ overnight. Next, the membranes were incubated with the HRP labeled second antibody (1:5000; Wuhan Boster Biological Technology, ltd., Wuhan, china) at $37^{\circ} \mathrm{C}$ for $1 \mathrm{~h}$. The ECL system (Pierce,
Table 1 The sequences of primers

\begin{tabular}{ll}
\hline Primers & Sequences(5'-3') \\
\hline NORAD-F & TGATAGGATACATCTTGGACATGGA \\
NORAD-R & AACCTAATGAACAAGTCCTGACATACA \\
OPG-F & TTGCACCACTCCAAATCCAG \\
OPG-R & AATCGCACCCACAACCG \\
RANK-F & GTCTCATCGTCCTGCTCCTCTT \\
RANK-R & CAGCGTTTTCCCTCCCTTC \\
RANKL-F & GCAGCATCGCTCTGTTCCTGTA \\
RANKL-R & GCATGAGTCAGGTAGTGCTTCTGTG \\
$\beta$-actin-F & AGACCACCTTCAACTCGATCAT \\
$\beta$-actin-R & ACTCGTCATACTCCTGCTTGCT \\
miR-26a-5p-F & CGCGAATTCTTGAGGTGAGGCTCAGGAGG \\
miR-26a-5p-R & ACGGGATCCTTGCTACAGGCAAAGGGT \\
U6-F & CTCGCTTCGGCAGCAGCACATATA \\
U6-R & AAATATGGAACGCTTCACGA \\
\hline
\end{tabular}

Rockford, IL, USA) was used to visualize the protein bands, and image lab version 3.0 (Bio-Rad Laboratories, Inc.) was used to analyze the intensity of the bands.

\section{Dual-luciferase reporter gene assay}

The potential binding site between NORAD and miR26a-5p was predicted by StarBase (http://starbase.sysu. edu.cn/index.php), and relevant primers (NORAD-MT and NORAD-WT) were synthesized by Shanghai GenePharma. HEK293 cells were seeded in 96-well plates until grew into about $85 \%$ concentration, miR-26a-5p mimics, mimics NC, NORAD-MT, or NORAD-WT were co-transfected into cells by the Lipofectamine 2000 reagent. After transfection for $48 \mathrm{~h}$, Dual-Luciferase Reporter Gene Assay Kit (Beyotime) was used to detect the relative fluorescence intensity.

\section{Cell proliferation assay}

The proliferation ability of hBMSCs was measured by the Cell Counting Kit-8 (KeyGEN Biotech Inc., Shanghai, China) assay. Briefly, BMSCs $(3 \times 104 \mathrm{cell} / \mathrm{ml})$ were seeded in 96-well plates after corresponding treatment. After incubation for $0,24,48$, and $72 \mathrm{~h}$, cells were incubated with $10 \mu \mathrm{l} \mathrm{CCK-8} \mathrm{solution} \mathrm{for} 2 \mathrm{~h}$ at $37^{\circ} \mathrm{C}$ and $5 \% \mathrm{CO}_{2}$. Subsequently, the optical density (OD) value at $490 \mathrm{~nm}$ was measured by a spectrophotometric microplate reader (Bio-Rad, Hercules, CA,USA).

\section{Cell apoptosis assay}

Cell apoptosis was estimated by Annexin V-FITC/PI Apoptosis Assay Kit (Sangon Biotech, Shanghai, China). After corresponding treatment, BMSCs were incubated with $10 \mu \mathrm{l}$ Annexin V-FITC and $5 \mu \mathrm{l}$ propidium iodide (PI) for $30 \mathrm{~min}$ in the dark. Then, the flow cytometry 
(BD Biosciences, Franklin Lakes, NJ, USA) was used to analyze cell apoptosis.

\section{Statistical analysis}

All experimental data were expressed as mean \pm standard deviation (SD), and GraphPad Prism 7.0 software (USA) was used to perform statistical analysis. One-way ANOVA was used to analyze the statistical differences among three or more groups. Unpaired Student's $t$ test was used to analyze the statistical differences between two groups. $P$ value less than 0.05 was considered as statistically significant.

\section{Results}

LncRNA NORAD expression is downregulated in SONFH tissues and Dex-treated hBMSCs, and treatment of Dex inhibits proliferation and the OPG/RANK/RANKL pathway in hBMSCs

We investigated the NORAD expression in bone marrow samples from patients with SONFH and femoral neck fracture (control) by RT-qPCR. As shown in Fig. 1a, NORAD expression in SONFH was lower than that in control group $(P<0.01)$. Next, we investigated the effects of Dex $\left(0,10^{-6}, 10^{-7}\right.$, and $\left.10^{-8} \mathrm{M}\right)$ on NORAD expression, proliferation, and OPG/RANK/RANKL pathway in hBMSCs. As presented in Fig. 1b, Dex reduced NORAD expression in a dose-dependent manner. In addition,
Dex inhibited hBMSC proliferation $\left(\mathrm{OD}_{490}\right.$ value) by increase in concentration and the time of exposure (Fig. 1c, $P<0.01)$. The mRNA expression of OPG, RANK, and RANKL was also detected by RT-qPCR. The results showed that the mRNA expression of OPG was decreased, and the mRNA expression of RANK and RANK $\mathrm{L}$ was increased in a dose-dependent manner $(P<0.05$, Fig. 1d-f).

\section{Overexpression of NORAD improves Dex-induced inhibition of proliferation and differentiation, and promotion of apoptosis in hBMSCs}

To investigate the role of NORAD in Dex-induced hBMSCs' osteoblastic differentiation, proliferation, and apoptosis, NORAD was overexpressed in hBMSCs. The results of RT-qPCR showed that NORAD expression was significantly increased when cells were transfected with NORAD overexpression vector, which suggested the transfection was successful $(P<0.01$, Fig. $2 a)$. As shown in Fig. $2 b-f$, the protein expression of RUNX2 and OPG which was decreased by Dex was increased after NORAD overexpression $(P<0.01)$, while the protein expression of RANK and RANKL which was increased by Dex was reduced after NORAD overexpression $(P<0.01)$. Moreover, when NORAD was overexpressed, Dex-induced proliferation reduction was attenuated $(P<0.05$, Fig. 2 g). As can be seen in Fig. 2h, Dex increased apoptotic rate compared

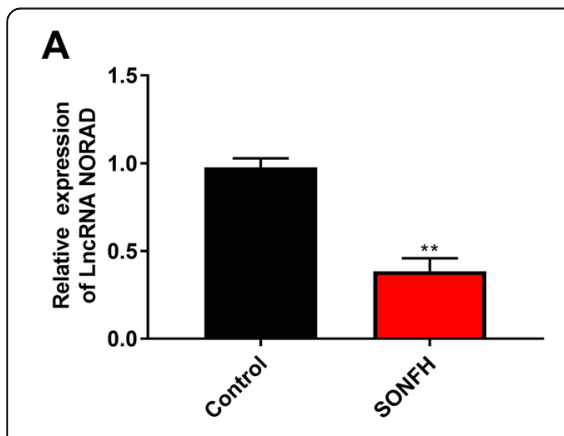

D

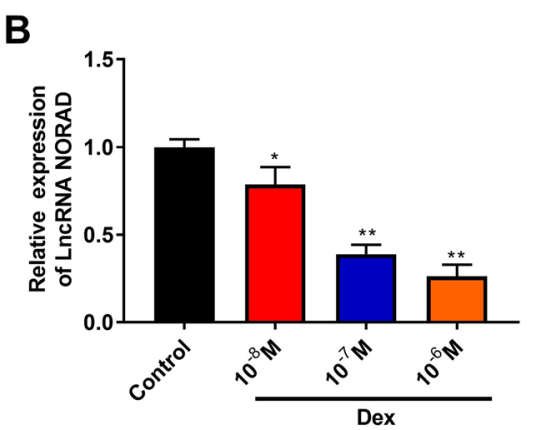

E

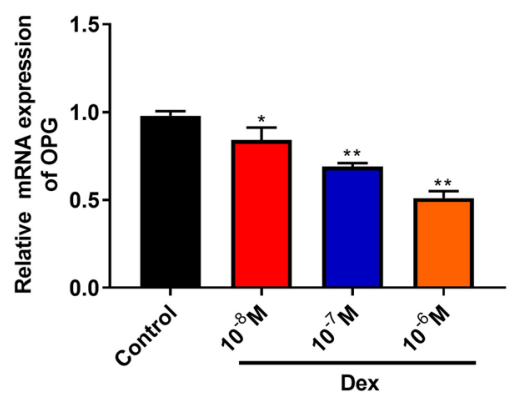

C
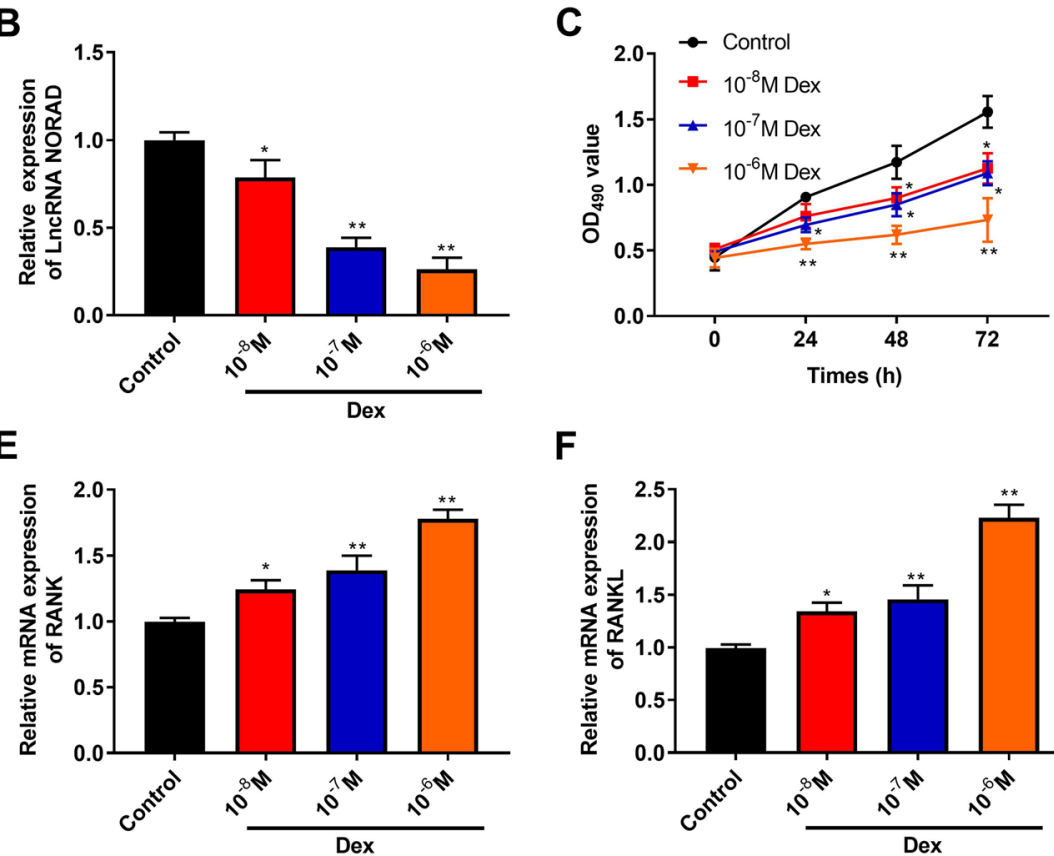

$\mathbf{F}$

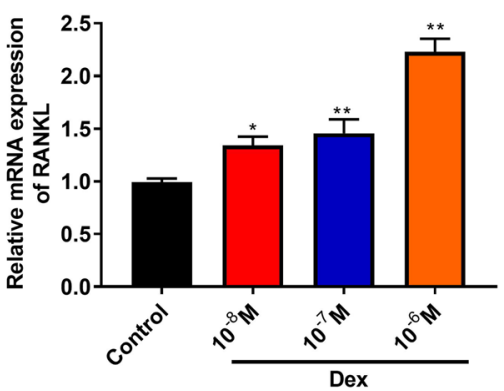

Fig. 1 LnCRNA NORAD expression was downregulated in SONFH tissues and Dex-treated hBMSCs, and treatment of Dex inhibited proliferation and the OPG/RANK/RANKL pathway in hBMSCs. a The NORAD expression in bone marrow samples from patients with SONFH $(n=20)$ and femoral neck fracture (control, $n=20$ ) was detected by RT-qPCR. b The NORAD expression in different concentration of Dex-treated hBMSCs was detected by RT-qPCR. $\mathbf{c}$ The proliferation ability of hBMSCs was measured by the CCK- 8 assay. $\mathbf{d}-\mathbf{f}$ The mRNA expression of OPG (d), RANK (e), and RANKL (f) was detected by RT-qPCR. $P<0.01$ and $P<0.05$ compared with the control group 


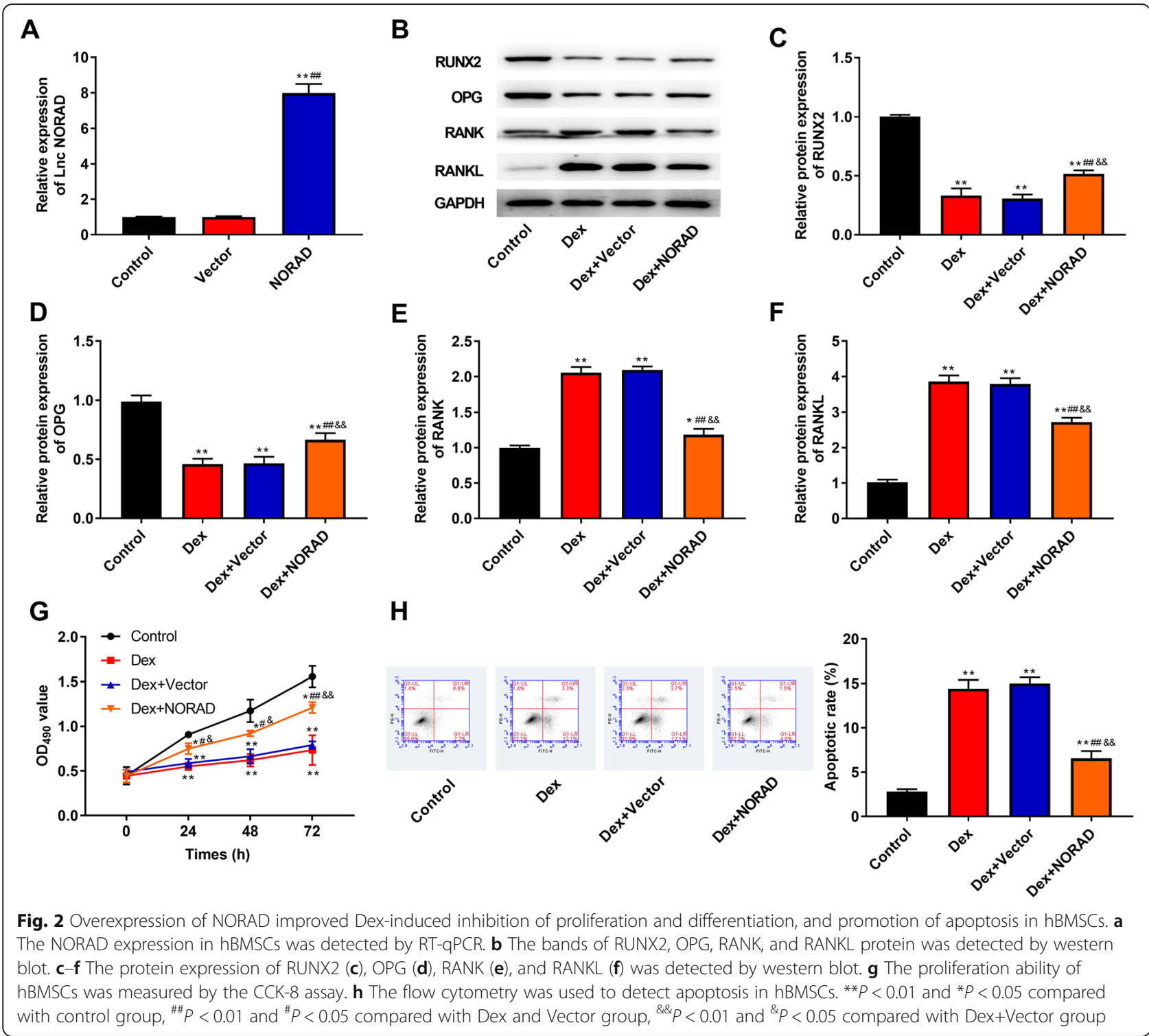

with control group $(P<0.01)$, but Dex-induced apoptosis increase was attenuated by overexpression of NORAD $(P<0.01)$.

Knockdown of NORAD aggravates Dex-induced inhibition of proliferation and differentiation, and promotion of apoptosis in hBMSCs

We hypothesized that knockdown of NORAD aggravated Dex-induced inhibition of proliferation and promotion of apoptosis in hBMSCs. To confirm this hypothesis, NORAD was low expressed by transfection with siRNA-NORAD in hBMSCs. As shown in Fig. 3a, NORAD expression in siRNA1-NORAD and siRNA2NORAD group was significantly decreased compared with Control and si-NC group $(P<0.01)$, which suggested the transfection was successful. Additionally, knockdown of NORAD significantly decreased the protein expression of RUNX2 and OPG decreased by Dex $(P<0.05$, Fig. $3 \mathrm{~b}-\mathrm{d})$, but knockdown of NORAD significantly increased the protein expression of RANK and RANKL increased by Dex $(P<0.01$, Fig. 3b, e, f). By knockdown of NORAD, proliferation was reduced compared with Dex and Dex + si-NC group $(P<0.05$, Fig. $3 g)$. In addition, Dex-induced apoptotic rate increase was aggravated by siRNA1-NORAD transfection in hBMSCs $(P<0.01$, Fig. 3h).

miR-26a-5p is a target and is negatively regulated by NORAD

In order to explore the mechanisms of NORADmediated progression in SONFH, we used StarBase to predict the downstream targets of NORAD. The results 


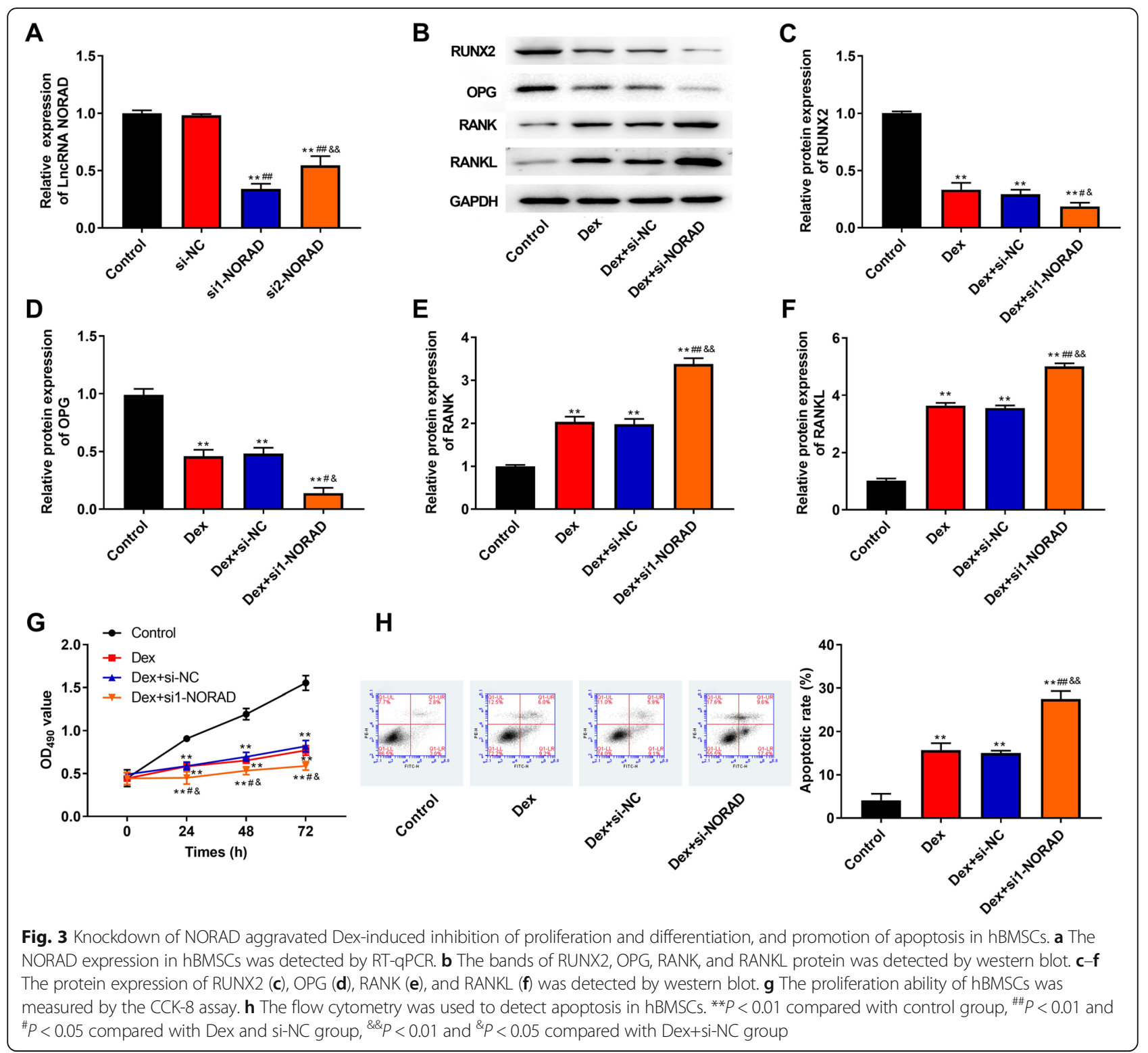

showed that NORAD might have direct effect on miR26a-5p (Fig. 4a). Next, dual-luciferase reporter gene assay was performed to further confirm the prediction. As shown in Fig. 4b, when HEK293 cells were cotransfected with miR-26a-5p mimics and NORAD-WT, the luciferase activity was remarkably reduced compared with co-transfection of NORAD-WT and miR-145 mimics $\mathrm{NC}$, while there were no significant difference in NORAD-MT, which was indicated that miR-26a-5p was a target of NORAD. In addition, miR-26a-5p expression in SONFH was higher than that in Control group $(P<$ 0.01 , Fig. 4c), and NORAD and miR-26a-5p expression represented a negative correlation $(P<0.01$, Fig. $4 d)$. Moreover, it was found that miR-26a-5p was downregulated in hBMSCs transfected with NORAD overexpression vector $(P<0.01$, Fig. 4e), while knockdown of NORAD led to the opposite results.

NORAD improves Dex-induced inhibition of proliferation and differentiation, and promotion of apoptosis by regulation of miR-26a-5p in hBMSCs

To investigate whether NORAD exerted its effects by regulating miR-26a-5p, NORAD overexpression vector and miR-26a-5p mimics were co-transfected into hBMSCs. As shown in Fig. 5a, miR-26a-5p expression was increased when cells were transfected with miR-26a$5 \mathrm{p}$ mimics, which suggested the transfection was successful $(P<0.01)$. As illustrated in Fig. $5 \mathrm{~b}-\mathrm{f}$, the protein expression of RUNX2 and OPG was increased and the protein expression of RANK and RANKL was decreased 


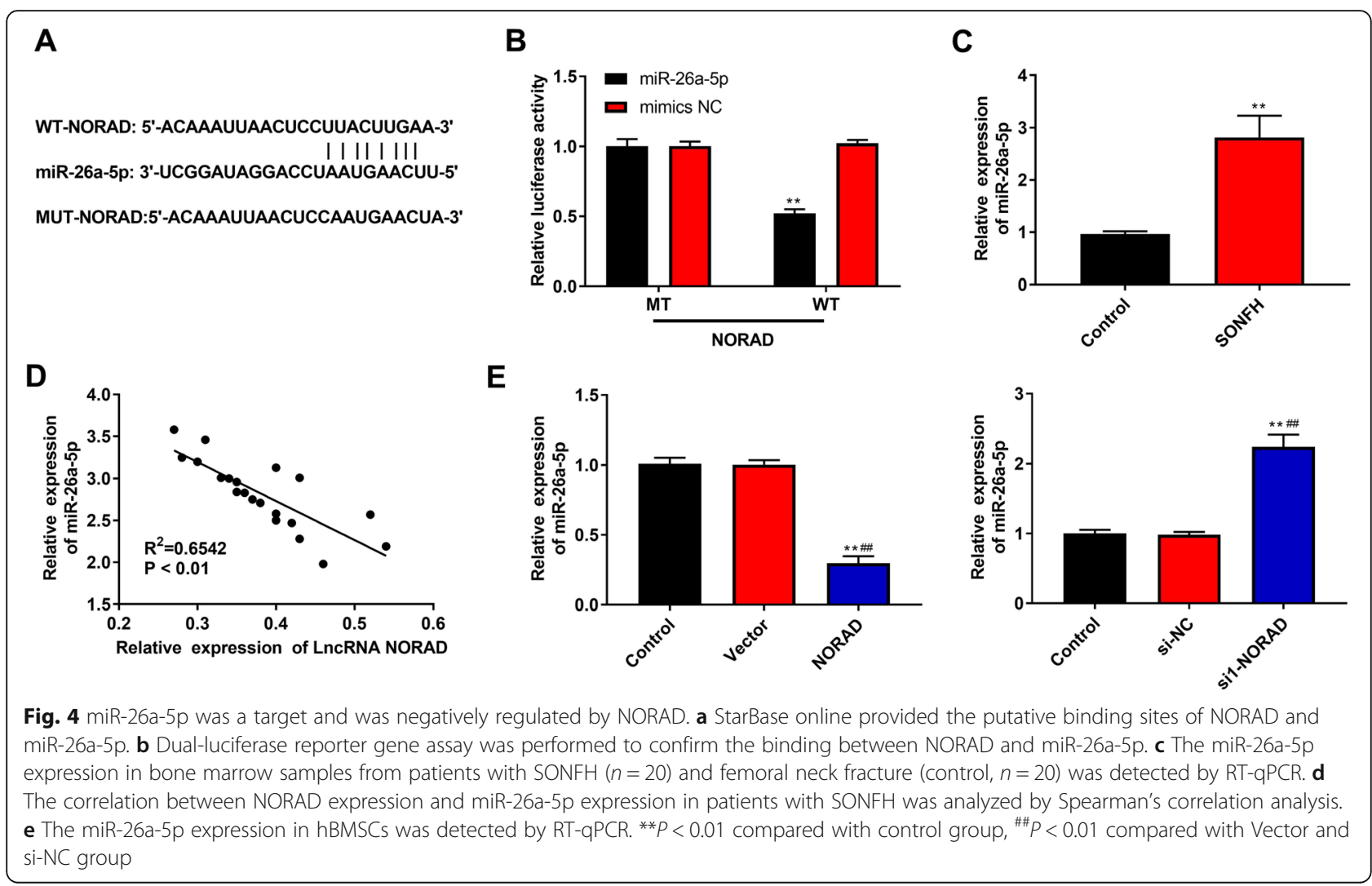

by overexpression of NORAD $(P<0.01)$, while transfection of miR-26a-5p mimics remarkably inhibited these effects $(P<0.01)$. In addition, as can be seen in Fig. $5 \mathrm{~g}$, $h$, proliferation ability was greatly enhanced but cell apoptosis was repressed by overexpression of NORAD $(P<0.05)$, while these effects were weakened by overexpression of miR-26a-5p $(P<0.05)$. Taken together, the above findings suggested that NORAD improved Dexinduced inhibition of proliferation and differentiation, and promotion of apoptosis by regulation of miR-26a-5p in hBMSCs.

\section{Discussion}

Bone cells' death caused by blood circulation disorders lead to the occurrence of ONFH [28]. ONFH is divided into traumatic and non-traumatic, and the use of longterm high-dose GC caused most non-traumatic necrosis [1]. Recently, many studies have shown that BMSCs play an important role in the development of ONFH $[29,30]$. Meanwhile, more and more evidences have showed that various lncRNAs and miRNAs have regulatory effects in the progress of the ONFH [31-33]. NORAD has been confirmed to be overexpressed in various cancers, and it is involved in proliferation, differentiation, and apoptosis $[16,34,35]$. In the present study, we found that NORAD expression was downregulated in SONFH tissues, while miR-26a-5p expression was upregulated. Overexpression of NORAD improved Dex-induced inhibition of proliferation and differentiation, and promotion of apoptosis in hBMSCs, while knockdown of NORAD led to the opposite results. Moreover, NORAD improved Dexinduced inhibition of proliferation and differentiation, and promotion of apoptosis by regulation of miR-26a-5p in hBMSCs.

The dysregulation of osteogenesis differentiation is an important pathway in SONFH; thus, BMSCs have become a research hotspot and are considered to be potential in treatment of SONFH [36]. Although some development of SONFH have made in clinical and basic researches, its molecular mechanisms are still unclear. In recent years, there are more and more report about the role of lncRNAs in osteogenesis. For example, Wei et al. [37] have demonstrated that HOTAIR expression is upregulated in samples of non-traumatic ONFH, and knockdown of HOTAIR promotes osteogenic differentiation and proliferation. Zhuang et al. [38] have found that MEG3 promotes osteogenic differentiation of MSCs in multiple myeloma. In our study, it was found that NORAD expression was downregulated in SONFH tissues. DEX could inhibit proliferation and induce apoptosis of BMSCs [39, 40]. We investigated the effects of $\operatorname{Dex}\left(0,10^{-6}, 10^{-7}\right.$, and $\left.10^{-8} \mathrm{M}\right)$ on NORAD expression and proliferation in hBMSCs and found that Dex reduced NORAD expression and inhibited cell proliferation in a dose-dependent 


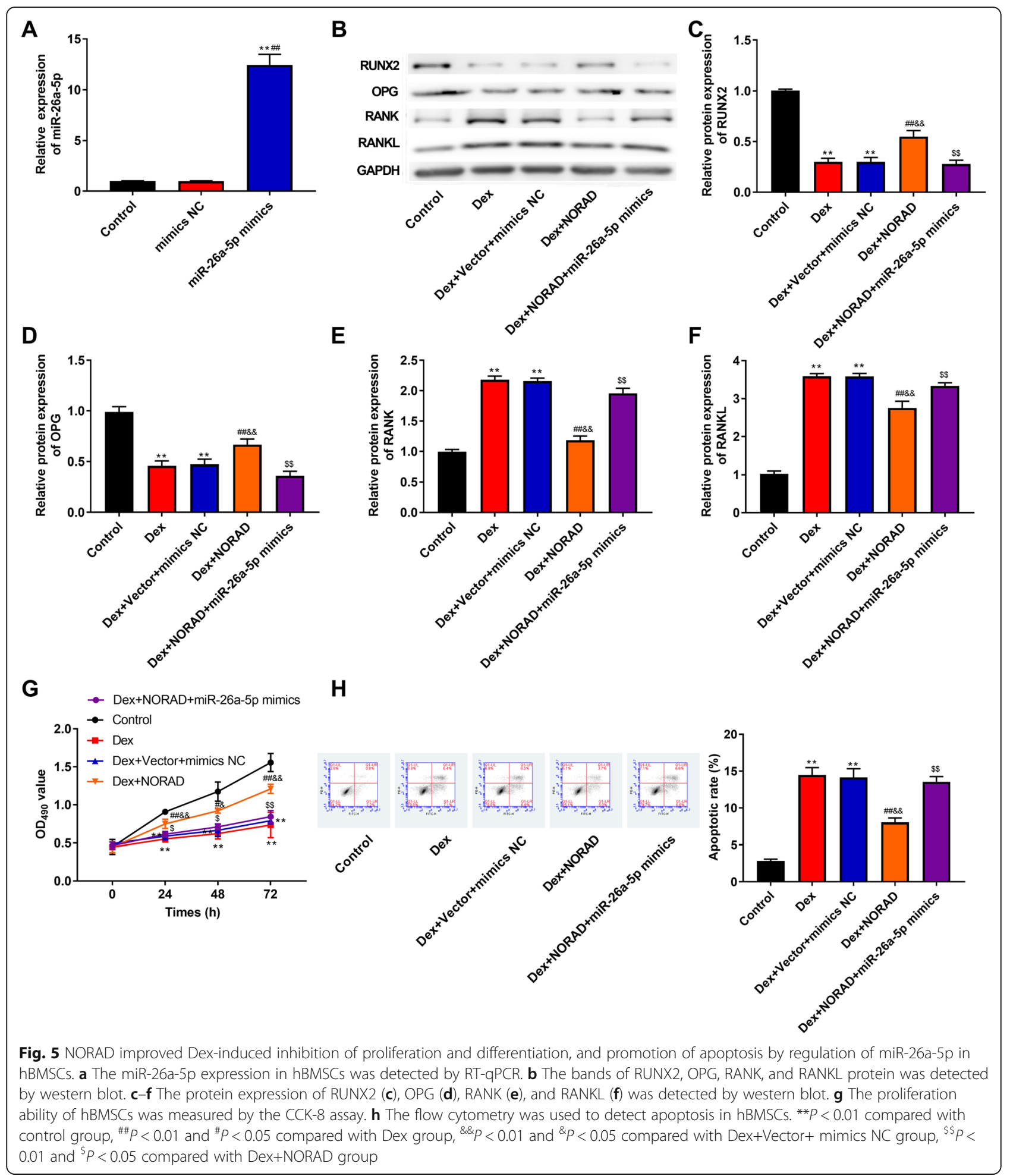

manner. In addition, we also found that overexpression of NORAD improved Dex-induced inhibition of proliferation and promotion of apoptosis in hBMSCs, while knockdown of NORAD led to the opposite results. In short, the above findings indicated that NORAD participated in the development of SONFH.

MicroRNAs (miRNAs, usually with 19-25 nucleotides) are small non-coding endogenous RNAs, which can bind 
to the 3'UTR of target mRNAs and change the stability of mRNA or protein translation, thus regulating various physiological process $[41,42]$. In recent decades, there are a lot of researches about the effects of miRNAs on the osteogenesis of MSCs. For instance, Zha et al. [43] have found that miR-34a reduces the inhibitory effects of Dex on mMSCs and osteoblasts. Jia et al. [44] have shown that miR-17-5p facilitates the proliferation and differentiation of HMSC-bm cells by targeting SMAD7. To further analyze the mechanism of NORAD in SONFH, StarBase predicted that NORAD and miR-26a$5 \mathrm{p}$ had a binding site. In addition, Luzi et al. [45] have found that miR-26a inhibits terminal differentiation of hADSCs. In this study, the luciferase activity was remarkably reduced; then, cells were co-transfected with miR-26a-5p mimics and NORAD-WT. In addition, miR26a-5p expression in SONFH was upregulated, and NORAD and miR-26a-5p expression represented a negative correlation. Moreover, NORAD promoted cell proliferation and inhibited cell apoptosis, while these effects were weakened by overexpression of miR-26a-5p. Taken together, these results indicated NORAD improved Dexinduced inhibition of proliferation and promotion of apoptosis by regulation of miR-26a-5p in hBMSCs.

Osteoprotegerin (OPG) is a member of tumor necrosis factor receptor family, which has the function of inhibiting osteoclast differentiation and increasing bone density [46]. OPG can directly bind to RANKL to inhibit the combination between RANKL and RANK, and thus inhibit the activation of osteoclast [47]. OPG/RANK/ RANKL together constitute a key signaling pathway in osteoclast differentiation and bone resorption [48]. In the present study, it was found that Dex reduced the mRNA expression of OPG and increased the mRNA expression of RANK and RANKL in a dose-dependent manner. In addition, overexpression of NORAD improved DEX-induced inhibition of OPG expression and promotion of RANK and RANKL expression, while these effects were weakened by overexpression of miR26a-5p. RUNX2, as a lineage-specific transcription factor, participates in tumor metastasis and osteogenesis [49-51]. Zhou et al. [52] have demonstrated that overexpression of NORAD increases the mRNA and protein expression of RUNX2, while knockdown of NORAD inhibits the RUNX2 expression in breast cancer cells. In our study, overexpression of NORAD significantly increased the protein expression of RUNX2, but knockdown of NORAD decreased the protein expression of RUNX2. Moreover, transfection of miR-26a-5p mimics remarkably reversed the increase of RUNX2 expression induced by overexpression of NORAD. All in all, the above results suggested that NORAD improved Dexinduced inhibition of differentiation by regulation of miR-26a-5p in hBMSCs.

\section{Conclusions}

NORAD expression was downregulated in SONFH tissues, while miR-26a-5p expression was upregulated. Moreover, NORAD improved Dex-induced inhibition of proliferation and differentiation, and promotion of apoptosis by regulation of miR-26a-5p in hBMSCs. This study may provide a new insight for the treatment and diagnosis of SONFH.

\section{Acknowledgements \\ Not applicable.}

\section{Authors' contributions}

Dapeng Fu designed the experiment; Sheng Yang obtained the data;

Jianmin Lu and Haoyi Lian analyzed the data; Kairong Qin wrote the paper;

Dapeng Fu reviewed the article critically. The authors read and approved the

final manuscript.

\section{Funding}

Not applicable,

\section{Availability of data and materials}

The datasets used and analyzed during the current study are available from the corresponding author on reasonable request.

\section{Ethics approval and consent to participate}

The protocol of this research has been approved by the Ethics Committee of Affiliated Zhongshan Hospital of Dalian University. All patients have signed written informed consent.

\section{Consent for publication}

Not applicable.

\section{Competing interests}

Not applicable.

\section{Author details}

${ }^{1}$ Department of Orthopaedics, Affiliated Zhongshan Hospital of Dalian University, Dalian 116001, Liaoning, People's Republic of China. ${ }^{2}$ Department of Biomedical Engineering, Dalian University of Technology, Dalian 116024, Liaoning, People's Republic of China.

Received: 22 September 2020 Accepted: 6 December 2020

Published online: 07 January 2021

\section{References}

1. Xie Y, Hu JZ, Shi ZY. MiR-181d promotes steroidinduced osteonecrosis of the femoral head by targeting SMAD3 to inhibit osteogenic differentiation of hBMSCs. Eur Rev Med Pharmacol Sci. 2018;22:4053-62.

2. Lamb JN, Holton C, O'Connor P, Giannoudis PV. Avascular necrosis of the hip. BMJ. 2019;365:12178.

3. Zhang $Y$, Yin J, Ding $H$, et al. Vitamin K2 prevents glucocorticoid-induced osteonecrosis of the femoral head in rats. Int J Biol Sci. 2016;12:347-58.

4. Assouline-Dayan. Pathogenesis and natural history of osteonecrosis. Semin Arthritis Rheumatism. 2002;32:94-124

5. Zhao DW, Yu M, Hu K, et al. Prevalence of nontraumatic osteonecrosis of the femoral head and its associated risk factors in the Chinese population: results from a nationally representative survey. Chin Med J. 2015;128:2843-50.

6. Murata M, Kumagai K, Miyata N, Osaki M, Shindo H. Osteonecrosis in strokeprone spontaneously hypertensive rats: effect of glucocorticoid. J Orthop Sci. 2007;12:289-95.

7. Lee JS, Roh HL, Kim CH, Jung JS, Suh KT. Alterations in the differentiation ability of mesenchymal stem cells in patients with nontraumatic osteonecrosis of the femoral head: comparative analysis according to the risk factor. J Orthop Res. 2006;24:604-9.

8. Seo BM, Miura M, Gronthos S, et al. Investigation of multipotent postnatal stem cells from human periodontal ligament. Lancet. 2004;364:149-55.

9. Dong $\mathrm{CH}$, Deng YS, Yang XJ, et al. The interplay of transcriptional and posttranscriptional regulation of migration of mesenchymal stem cells during 
early stages of bone fracture healing. Eur Rev Med Pharmacol Sci. 2017;21: $5542-7$.

10. Shi S, Wu X, Wang $X$, et al. Differentiation of bone marrow mesenchymal stem cells to cardiomyocyte-like cells is regulated by the combined low dose treatment of transforming growth factor-beta1 and 5-azacytidine. Stem Cells Int. 2016:3816256:30.

11. Sun Z, Yang S, Ye S, et al. Aberrant CpG islands' hypermethylation of ABCB1 in mesenchymal stem cells of patients with steroid-associated osteonecrosis. J Rheumatol. 2013;40:1913-20.

12. Li J, Xu X, Wei C, Liu L, Wang T. Long noncoding RNA NORAD regulates lung cancer cell proliferation, apoptosis, migration, and invasion by the miR30a-5p/ADAM19 axis. Int J Clin Exp Pathol. 2020;13:1-13.

13. Huang $G$, Kang $Y$, Huang $Z$, et al. Identification and characterization of long non-coding RNAs in osteogenic differentiation of human adipose-derived stem cells. Cell Physiol Biochem. 2017:42:1037-50.

14. Wang $Y$, Song $X$, Li Z, Liu B. Long non-coding RNAs in coronary atherosclerosis. Life Sci. 2018;211:189-97.

15. Indolfi C, laconetti C, Gareri C, Polimeni A, De Rosa S. Non-coding RNAs in vascular remodeling and restenosis. Vasc Pharmacol. 2019;114:49-63.

16. Huo H, Tian J, Wang R, et al. Long non-coding RNA NORAD upregulate SIP1 expression to promote cell proliferation and invasion in cervical cancer. Biomed Pharmacother. 2018;106:1454-60.

17. Miao Z, Guo X, Tian L. The long noncoding RNA NORAD promotes the growth of gastric cancer cells by sponging miR-608. Gene. 2019;687:116-24.

18. Zhang J, Li XY, Hu P, Ding YS. IncRNA NORAD contributes to colorectal cancer progression by inhibition of miR-202-5p. Oncol Res. 2018;26:1411-8.

19. Xu C, Zhu LX, Sun DM, Yao H, Han DX. Regulatory mechanism of IncRNA NORAD on proliferation and invasion of ovarian cancer cells through miR199a-3p. Eur Rev Med Pharmacol Sci. 2020:24:1672-81.

20. Wang $\mathrm{X}$, Zou J, Chen $\mathrm{H}$, et al. Long noncoding RNA NORAD regulates cancer cell proliferation and migration in human osteosarcoma by endogenously competing with miR-199a-3p. IUBMB Life. 2019;71:1482-91.

21. Tao W, Li Y, Zhu M, Li C, Li P. LncRNA NORAD promotes proliferation and inhibits apoptosis of gastric Cancer by regulating miR-214/Akt/mTOR Axis. Onco Targets Ther. 2019;12:8841-51.

22. Bian $W$, Jing $X$, Yang $Z$, et al. Downregulation of LncRNA NORAD promotes Ox-LDL-induced vascular endothelial cell injury and atherosclerosis. Aging. 2020;12:6385-400.

23. Jalali S, Bhartiya D, Lalwani MK, Sivasubbu S, Scaria V. Systematic transcriptome wide analysis of IncRNA-miRNA interactions. Plos One. 2013;8:6

24. Mao Y, Liu R, Zhou H, et al. Transcriptome analysis of miRNA-IncRNA-mRNA interactions in the malignant transformation process of gastric cancer initiation. Cancer Gene Ther. 2017;24:267-75.

25. Bian $E B$, Xiong ZG, Li J. New advances of IncRNAs in liver fibrosis, with specific focus on IncRNA-miRNA interactions. J Cell Physiol. 2019;234:2194-203.

26. Zhang W, Dong R, Diao S, et al. Differential long noncoding RNA/mRNA expression profiling and functional network analysis during osteogenic differentiation of human bone marrow mesenchymal stem cells. Stem Cell Res Ther. 2017:8:017-0485.

27. Li S, Hu C, Li J, et al. Effect of miR-26a-5p on the Wnt/Ca2+pathway and osteogenic differentiation of mouse adipose-derived mesenchymal stem cells. Calcif Tissue Int. 2016;99:174-86.

28. Feng $\mathrm{Y}$, Yang $\mathrm{SH}$, Xiao BJ, et al. Decreased in the number and function of circulation endothelial progenitor cells in patients with avascular necrosis of the femoral head. Bone. 2010;46:32-40.

29. Liu B, Yang F, Wei X, et al. An exploratory study of articular cartilage and subchondral bone reconstruction with bone marrow mesenchymal stem cells combined with porous tantalum/Bio-Gide collagen membrane in osteonecrosis of the femoral head. Mater Sci Eng C Mater Biol Appl. 2019; 99:1123-32.

30. Yu Y, Newman $H$, Shen $L$, et al. Glutamine metabolism regulates proliferation and lineage allocation in skeletal stem cells. Cell Metab. 2019; 29:966-78.

31. Xu Y, Jiang Y, Wang Y, et al. LINC00473 regulated apoptosis, proliferation and migration but could not reverse cell cycle arrest of human bone marrow mesenchymal stem cells induced by a high-dosage of dexamethasone. Stem Cell Res. 2020;48:11.

32. Xiang S, Li Z, Weng X. The role of IncRNA RP11-154D6 in steroid-induced osteonecrosis of the femoral head through BMSC regulation. J Cell Biochem. 2019:120:18435-45.
33. Liao W, Ning $Y, X u H J$, et al. BMSC-derived exosomes carrying microRNA122-5p promote proliferation of osteoblasts in osteonecrosis of the femoral head. Clin Sci. 2019;133:1955-75.

34. Zhao W, Wang L, Xu F. LncRNA NORAD stimulates proliferation and migration of renal cancer via activating the miR-144-3p/MYCN axis. Eur Rev Med Pharmacol Sci. 2020;24:10426-32.

35. Wan Y, Yao Z, Chen W, Li D. The IncRNA NORAD/miR-520a-3p facilitates malignancy in non-small cell lung cancer via PI3k/Akt/mTOR signaling pathway. Onco Targets Ther. 2020;13:1533-44.

36. Huang XZ, Huang J, Li WZ, et al. LnCRNA-MALAT1 promotes osteogenic differentiation through regulating ATF4 by sponging miR-214: implication of steroid-induced avascular necrosis of the femoral head. Steroids. 2020;154:31.

37. Wei B, Wei W, Zhao B, Guo X, Liu S. Long non-coding RNA HOTAIR inhibits miR-17-5p to regulate osteogenic differentiation and proliferation in nontraumatic osteonecrosis of femoral head. PLoS One. 2017;12:e0169097.

38. Zhuang W, Ge X, Yang S, et al. Upregulation of IncRNA MEG3 promotes osteogenic differentiation of mesenchymal stem cells from multiple myeloma patients by targeting BMP4 transcription. Stem Cells. 2015;33: 1985-97.

39. Chen F, Zhang L, OuYang Y, et al. Glucocorticoid induced osteoblast apoptosis by increasing E4BP4 expression via up-regulation of Bim. Calcif Tissue Int. 2014;94:640-7.

40. Fan Q, Zhan X, Li X, Zhao J, Chen Y. Vanadate inhibits dexamethasoneinduced apoptosis of rat bone marrow-derived mesenchymal stem cells. Ann Clin Lab Sci. 2015;45:173-80.

41. Sriram M, Sainitya R, Kalyanaraman V, Dhivya S, Selvamurugan N. Biomaterials mediated microRNA delivery for bone tissue engineering. Int J Biol Macromol. 2015;74:404-12.

42. Martin EC, Qureshi AT, Dasa V, et al. MicroRNA regulation of stem cell differentiation and diseases of the bone and adipose tissue: perspectives on miRNA biogenesis and cellular transcriptome. Biochimie. 2016;124:98-111.

43. Zha X, Sun B, Zhang R, et al. Regulatory effect of microRNA-34a on osteogenesis and angiogenesis in glucocorticoid-induced osteonecrosis of the femoral head. J Orthop Res. 2018;36:417-24.

44. Jia J, Feng X, Xu W, et al. MiR-17-5p modulates osteoblastic differentiation and cell proliferation by targeting SMAD7 in non-traumatic osteonecrosis. Exp Mol Med. 2014;25:43.

45. Luzi E, Marini F, Sala SC, et al. Osteogenic differentiation of human adipose tissue-derived stem cells is modulated by the miR-26a targeting of the SMAD1 transcription factor. J Bone Miner Res. 2008;23:287-95.

46. Geusens P. The role of RANK ligand/osteoprotegerin in rheumatoid arthritis, Ther Adv Musculoskelet Dis. 2012;4:225-33.

47. Martin TJ, Sims NA. RANKL/OPG; critical role in bone physiology. Rev Endocr Metab Disord. 2015;16:131-9.

48. Wittrant $Y$, Theoleyre S, Couillaud $S$, et al. Relevance of an in vitro osteoclastogenesis system to study receptor activator of NF-kB ligand and osteoprotegerin biological activities. Exp Cell Res. 2004;293:0-301.

49. Ge C, Zhao G, Li Y, et al. Role of Runx2 phosphorylation in prostate cancer and association with metastatic disease. Oncogene. 2016;35:366-76.

50. Guo ZJ, Yang L, Qian F, Wang YX, Bian X-W. Transcription factor RUNX2 upregulates chemokine receptor $C X C R 4$ to promote invasive and metastatic potentials of human gastric cancer. Oncotarget. 2016;7:20999-1012.

51. Akiyama Y, Hara K, Tajima T, Murota SI, Morita I. Effect of vitamin K2 (menatetrenone) on osteoclast-like cell formation in mouse bone marrow cultures. Eur J Pharmacol. 1994;263:0-185.

52. Zhou K, Ou Q, Wang G, et al. High long non-coding RNA NORAD expression predicts poor prognosis and promotes breast cancer progression by regulating TGF- $\beta$ pathway. Cancer Cell Int. 2019;19:019-0781.

\section{Publisher's Note}

Springer Nature remains neutral with regard to jurisdictional claims in published maps and institutional affiliations. 